\title{
Escala de Apoyo Social para cuidadores familiares de adultos mayores mexicanos
}

\author{
Social Support Scale for Family Caregivers of Elder Mexicans
}

Recibido: febrero 24 de 2012 | Revisado: mayo 16 de 2012 | Aceptado: julio 17 de 2012

\author{
Miriam Teresa Domínguez GuedeA* \\ María FERnANDA MANDUJANO JaQUEZ ** \\ MARCEla GeORgINA Quintero *** \\ TERESA IVETH SOTELO QUIÑÓNEZ **** \\ JosÉ CONCEPCIÓN GAXIOLA ROMERO ****** \\ Jesús ERNESTO VALENCIA MALDONADO ******* \\ Universidad de Sonora, México
}

doi:10.11144/Javeriana.UPSY12-2.easc

Para citar este artículo: Domínguez, M. T., Mandujano, M. F., Quintero, M. G., Sotelo, T. I., Gaxiola, J. C. \& Valencia, J. E. (2013). Escala de Apoyo Social para cuidadores familiares de adultos mayores mexicanos. Universitas Psychologica, 12(2), 391-402.

Universidad de Sonora, México. Profesora-investigadora del Dpto. de Psicología, líder del Cuerpo Académico Salud en Grupos Vulnerables, pertenece y a la Red Temática del Consejo Nacional de Ciencia y Tecnología "Envejecimiento, Salud y Desarrollo Social”. E-mail: miriamd@sociales. uson.mx

* Universidad de Sonora, México. Miembro del Equipo de Investigación y Acción para Cuidadores Familiares de Adultos Mayores del Cuerpo Académico Salud en Grupos Vulnerables. E-mail: maferm@gmail.com

*** Universidad de Sonora, México. Miembro del Equipo de Investigación y Acción para Cuidadores Familiares de Adultos Mayores del Cuerpo Académico Salud en Grupos Vulnerables. marcelag. quinterov@correoa.uson.mx

****** Universidad de Sonora, México. Profesora del Dpto. de Psicología y miembro del Equipo de Investigación y Acción para Cuidadores Familiares de Adultos Mayores del Cuerpo Académico Salud en Grupos Vulnerables. E-mail: tsotelo@psicom. uson.mx

**:***: Universidad de Sonora, México. Sistema Nacional de Investigadores nivel 1, actualmente profesorinvestigador del Departamento de Psicología. Email: joegaxiola@gmail.com

******** Universidad de Sonora, México. Miembro del Equipo de Investigación y Acción para Cuidadores Familiares de Adultos Mayores del Cuerpo Académico Salud en Grupos Vulnerables. E-mail: jevmac@gmail.com

\section{RES UMEN}

Mediante análisis factoriales exploratorios y confirmatorios, se validó la Escala de Apoyo Social para Cuidadores Familiares de Adultos Mayores. 23 ítems con cargas factoriales $\geq 0.51$, agrupados en cuatro factores explican $63 \%$ de la varianza del constructo, con valores de Alfa de Cronbach $\geq 0.85$; se demostró que el apoyo social tiene una relación causal positiva y significativa con apoyo material $(\beta=0.66)$, apoyo práctico $(\beta=0.69)$, apoyo emocional $(\beta=0.6)$ y apoyo de orientación $(\beta=0.81)$; los valores de ajuste del modelo son $\geq 0.97$. La escala validada puede ser aplicada junto con otras medidas que permitan valorar las necesidades que enfrentan las familias para generar acciones de apoyo dirigidas al adulto-mayor y a quien lo cuida. Palabras clave autores:

Apoyo social, adultos mayores, cuidadores.

Palabras clave adicionales:

Investigación cuantitativa, psicometría, validación, análisis factorial.

\footnotetext{
ABSTRACT

Using exploratory and confirmatory analysis factor, was validated the Social Support Scale for Family Caregivers of Older Adults. 23 items with factor loadings $\geq 0.51$, grouped into four factors it explain $63 \%$ of the variance of the construct, with Cronbach's alpha values $\geq 0.85$; was shown that social support has a positive and significant causal relationship with material support $(\beta=0.66)$, practical support $(\beta=0.69)$, emotional support $(\beta=0.6)$, and counseling support $(\beta=0.81)$, the model fit values was $\geq 0.97$. The validated scale can be applied together with other measures to assess needs faced by families to generate support actions aimed at adultsenior and caregiver.

Key words authors Social Support, Elder Adults, Caregivers.

Key words plus

Quantitative Research, Psychometry, Validation, Analysis Factor.
} 
Al cierre del siglo XX, la dinámica poblacional mexicana registró un incremento muy importante en el número de personas que alcanzaron los 60 años y más, previéndose también la tendencia del aumento en las décadas venideras. Dicho logro se ha visto ensombrecido por una situación que se presenta de manera acentuada en contextos sociales de precariedad económica, y es que con el repunte en el índice de envejecimiento, en nuestro país hay ahora toda una legión de personas debilitadas por la edad y por múltiples dependencias, urgiendo la atención a sus necesidades principalmente a nivel de salud y protección social (Gutiérrez-Robledo, Ávila-Fematt \& Montaña-Álvarez, 2010).

Son tres las instancias que en los países latinoamericanos intervienen en la oferta del cuidado: familia, Estado y mercado; en tanto que el mercado exige un poder adquisitivo suficiente y los sistemas públicos aún son limitados para atender las crecientes exigencias de la población envejecida, la familia es quien de manera protagónica atiende las demandas de cuidado de los mayores (Huenchuan \& Guzmán, 2007), implicando en la mayoría de los casos, francas repercusiones para la salud y el bienestar de las personas que asumen el cuidado de su familiar adulto mayor dependiente funcional (Lizárraga, Artetxe \& Posua, 2008; Ostwald, 2009; Rodríguez-Agudelo, Mondragón-Maya, Paz-Rodríguez, Chávez-Oliveros \& Solís-Vivanco, 2010). En estas circunstancias, la familia reduce al menos a la mitad los costos profesionales del cuidado (Spijker et al., 2009), tornándose en una alternativa muy conveniente para las instituciones, pero en tal proceso el propio cuidador se convierte en un paciente oculto (Molina, Iáñez \& Iáñez, 2005; Úbeda $\&$ Roca, 2008).

A pesar de su gran aportación en fuerza de trabajo y costos del cuidado, la familia aún no ha sido reconocida en México como sujeto de apoyo en términos de una política pública (Márquez-González, López, Romero-Moreno \& Losada, 2010) y el estudio de su problemática es aún poco documentado en publicaciones científicas. Dada esa situación, es fundamental dimensionar las necesidades de apoyo que tienen los cuidadores familiares de adultos mayores mexicanos, considerando al apoyo social como un tema de análisis obligado en un grupo que absorbe el impacto físico y económico derivado de la limitación que el Estado tiene para atender a su población envejecida, jugando así un papel crucial en la sociedad que, en el más justo de los casos, le debe correspondencia.

Las aproximaciones multidisciplinarias sobre salud tienen bien establecido que el apoyo social se asocia al bienestar de las personas (Gallegos-Carillo et al., 2009; Gracia \& Herrero, 2006; Kafetsios \& Sideridis, 2006; Méndez \& Pozo, 2011), tanto por sus propiedades estructurales como funcionales (Barrón \& Sánchez, 2001), identificándose mejores indicadores de salud física y mental en personas que tienen acceso a procesos de apoyo versus aquellas que se encuentran en situaciones de aislamiento social (Golden et al., 2009; Ostwald, 2009). Tales consideraciones se confirman en muestras de cuidadores, pues se ha encontrado que altos niveles de apoyo, se corresponden con mejores niveles salud y bienestar (Reid, Stajduhar \& Chappell, 2010; Steiner et al., 2008), mitiga la vivencia de emociones negativas (Crespo \& López, 2007) tales como la ira (Márquez-González et al., 2010), estrés (Betrabet, 2009; Lavela \& Ather, 2010), sobrecarga, depresión y ansiedad (Arango-Lasprilla, Moreno, Rogers \& Francis, 2009; Pearce \& Singer, 2006; RodríguezAgudelo et al., 2010). En suma, el apoyo social percibido por los cuidadores familiares de adultos mayores refleja la ayuda que este recibe y facilita el ajuste, aligera la carga objetiva y subjetiva de las demandas del cuidado, aumenta las experiencias emocionales satisfactorias, contribuyendo significativamente a su crecimiento personal (FernándezLansac \& Crespo, 2011).

Apoyo social es definido por Lin y Ensel (1989) como el proceso (de percepción o recepción) a través del cual los recursos de la estructura social permiten satisfacer necesidades, sean instrumentales y/o de expresión, en situaciones cotidianas o de crisis. El apoyo implica un flujo de recursos, acciones e información que se concreta en ayudas instrumentales, materiales, emocionales y de orientación (Guzmán, Huenchuan \& Montes de Oca, 2003), siendo todas ellas relevantes para quien cuida de un familiar adulto mayor, que diariamente encara 
necesidades emocionales y conflictos de relación interpersonal, carencias económicas, dificultades instrumentales y falta de orientación para responder a la multiplicidad de demandas que genera el cuidado (Domínguez-Guedea, Damacena et al., 2009).

Dada su conceptualización multidimensional, existe una dificultad para medir el apoyo social atendiendo por completo a su complejidad, de forma que se pueden considerar variables del receptor de apoyo, del proveedor, de la interacción entre ambos, la correspondencia entre el apoyo proveído y la necesidad de este, así como la pertinencia temporal del apoyo, entre otros (Hupcey, 1998). Dicha complejidad se ha traducido en diversas estrategias para operacionalizar el apoyo social que reciben los cuidadores familiares, encontrando en la literatura internacional autores que recurren al uso de instrumentos psicométricos diseñados para medir el constructo en población general (ej., ArangoLasprilla et al., 2009), emplean medidas generales de apoyo pero que son validadas en sus muestras de cuidadores (ej. Crespo \& López, 2007; Hudson \& Hayman-White, 2006; Marziali, McCleary \& Streiner, 2010; Monahan \& Hooker, 1997; Spaid $\&$ Barusch, 1994; Wilks, 2009), medidas diseñadas para poblaciones específicas, tales como pacientes crónicos o adultos mayores (ej., Márquez-González et al., 2010; Rapp, Shumaker, Schmidt, Naughton \& Anderson, 1998; Van Den Wijngaart, VernooijDassen \& Felling, 2007) aplicadas en cuidadores

\section{TABLA 1}

Caracterización de la muestra

\begin{tabular}{|c|c|c|}
\hline Variables & Categorías de clasificación & Respuesta \% \\
\hline Sexo & $\begin{array}{l}\text { Femenino } \\
\text { Masculino } \\
\end{array}$ & $\begin{array}{c}90.4 \\
9.6\end{array}$ \\
\hline Situación conyugal & $\begin{array}{l}\text { No tiene pareja } \\
\text { Sí tiene pareja }\end{array}$ & $\begin{array}{l}42 \\
58 \\
\end{array}$ \\
\hline Grado de escolaridad & $\begin{array}{c}\text { Hasta secundaria } \\
\text { Preparatoria/carrera técnica } \\
\text { Licenciatura/posgrado }\end{array}$ & $\begin{array}{l}30.6 \\
32.5 \\
36.6\end{array}$ \\
\hline Tiene trabajo remunerado & $\begin{array}{l}\text { No } \\
\text { Sí }\end{array}$ & $\begin{array}{l}53.1 \\
46.9 \\
\end{array}$ \\
\hline Ocupación principal & $\begin{array}{c}\text { Hogar } \\
\text { Estudiante } \\
\text { Pensionado/a } \\
\text { Trabajador/a independiente } \\
\text { Trabajador/a asalariado/a } \\
\text { Desempleado/a } \\
\text { No respondieron }\end{array}$ & $\begin{array}{c}37.5 \\
2.7 \\
6.2 \\
15.2 \\
33.9 \\
1.8 \\
2.7\end{array}$ \\
\hline Ingreso mensual familiar & $\begin{array}{l}\text { Menos de } 9000 \text { pesos } \\
\text { Más de } 9000 \text { pesos }\end{array}$ & $\begin{array}{l}52.8 \\
47.2\end{array}$ \\
\hline Parentesco con el adulto mayor cuidado & $\begin{array}{c}\text { Hijo } \\
\text { Esposo/a } \\
\text { Nieto/a } \\
\text { Hermano/a } \\
\text { Mamá } \\
\text { Otros familiares(nueras, sobrinos, } \\
\text { primos, tíos, cuñada) }\end{array}$ & $\begin{array}{c}79.2 \\
6.4 \\
4.2 \\
3.4 \\
0.8 \\
6\end{array}$ \\
\hline Vive con el adulto mayor & $\begin{array}{l}\text { No } \\
\text { Sí }\end{array}$ & $\begin{array}{l}30.7 \\
69.3 \\
\end{array}$ \\
\hline Sexo del adulto mayor cuidado & $\begin{array}{l}\text { Femenino } \\
\text { Masculino }\end{array}$ & $\begin{array}{c}193 \\
70\end{array}$ \\
\hline Edad del adulto mayor cuidado & $\begin{array}{l}\text { 60-76 años } \\
77-85 \text { años } \\
86-99 \text { años }\end{array}$ & $\begin{array}{c}99 \\
103 \\
62\end{array}$ \\
\hline
\end{tabular}

Fuente: elaboración propia. 
familiares y conjuntos de preguntas que no forman parte de una escala pero que exploran los contenidos de interés del investigador (ej., Lee, 2008).

A pesar de los avances de esta área de investigación y con una situación social crítica, en México aún se carece de un instrumento que haya probado ser válido y confiable para medir el apoyo social entre cuidadores familiares de adultos mayores, razón por la cual el objetivo del presente estudio fue validar de manera confirmatoria la Escala de Apoyo Social en Cuidadores Familiares de Adultos Mayores (EP/ CFAM) en un contexto sociocultural mexicano.

\section{Método}

\section{Participantes}

Mediante un muestreo no probabilístico y por conveniencia, se colectaron datos de 265 personas que fungen como cuidadores de un adulto mayor dependiente funcional. Los criterios de inclusión fueron: a) ser familiar del receptor de cuidados; b) vivir con el adulto mayor o bien visitarlo al menos una vez por semana y c) para ser considerado cuidador, el participante tenía que ser responsable de proveer asistencia al adulto mayor al menos en una de las actividades indicadas en el índice de dependencia funcional referido en el apartado de instrumentos. Se excluyeron los casos que no completaron el $80 \%$ o más de los ítems del conjunto de instrumentos aplicados.

Las edades de los participantes variaron de los 19 a los 82 años $(M=50 ; D E=13.15)$ y las edades de los adultos mayores que reciben los cuidados fluctuaron entre los 60 y los 98 años $(M=78.4 ; D E=9.1)$; el tiempo de cuidado del adulto mayor varió de 2 meses a 60 años, registrándose un promedio de ocho años fungiendo como cuidador. En la Tabla 1 se exponen mayores detalles de la caracterización de la muestra participante.

\section{Materiales}

Para identificar las actividades en las cuales el adulto mayor es dependiente del cuidador, se aplicó un formulario que integra dos índices de valoración geriátrica, cuyas versiones originales fueron modificadas por Domínguez-Guedea y López-Dávalos (2008) con base en la propuesta de Shah, Vanclay y Cooper (1989), para los fines de la investigación general de la cual se deriva el presente estudio; esos índices son el Barthel Index, original de Mahoney y Barthel (1965) y el Instrumental Activities of Daily Living Scale (Lawton \& Brody, 1969). La autora agregó ítems sobre la capacidad del adulto mayor para comunicarse y mantener relaciones sociales. En total, el instrumento consta de 30 reactivos que valoran el nivel de ayuda que requiere el adulto mayor para desempeñar las actividades básicas, instrumentales y de comunicación. El instrumento es respondido por el cuidador utilizando las siguientes opciones de respuesta: 1) no necesita ayuda; 2) lo hace con ayuda de alguien; 3) depende totalmente de alguien. Se aplicó también una ficha de datos sociodemográficos del respondiente, así como datos generales del adulto mayor que recibe los cuidados.

La medida que se debía validar fue la Escala de Apoyo Social en Cuidadores Familiares de Adultos Mayores (EAP/CFAM), diseñada y validada por Domínguez-Guedea (2005) y Domínguez-Guedea, Tróccoli et al. (2009) en cuidadores familiares de adultos mayores en la ciudad de Brasilia, Brasil. Para su aplicación en el contexto mexicano se hizo la traducción (al portugués) y re-traducción (al español), y después fue sometida a un análisis de jueces y pilotaje para constatar la pertinencia teórica del conjunto de ítems, así como la comprensión semántica de la escala en el nuevo contexto cultural, realizándose los ajustes necesarios.

La EAP/CFAM quedó conformada por 23 ítems tipo Likert de cuatro puntos que miden la percepción de apoyo de acuerdo al parámetro de frecuencia $(1=$ nunca, $2=$ pocas veces, $3=$ muchas veces, 4 = siempre). Las áreas de apoyo exploradas son: Apoyo Material (siete ítems), Apoyo Práctico (cinco ítems), Apoyo Emocional (seis ítems) y Apoyo de Orientación (cinco ítems). La EAP/CFAM incluye también cinco preguntas tipo Likert de cinco puntos que evalúa la satisfacción del respondiente en cada área de apoyo $(1=$ nada satisfecho; $5=$ muy satisfecho). Como parte de los parámetros estructurales del apoyo social se mide el tamaño de la red 
social (sumando el número de personas de quienes el cuidador considera recibir apoyo) y las fuentes de apoyo (familiares, amigos, profesionales, etc.).

\section{Procedimiento}

El procedimiento para identificar a las personas que cumplían los criterios de participación en el estudio se realizó a través de dos tipos de contacto: a) el realizado con instituciones que prestan servicios a adultos mayores y b) el efectuado a través de redes personales.

Se denominó contacto institucional cuando los participantes accedieron a colaborar con el proyecto una vez que se practicó un protocolo de información con los directores o responsables de las unidades de salud del primer, segundo y tercer nivel de atención (clínicas comunitarias, hospitales generales y centros de salud mental), organizaciones de la sociedad civil, grupos de pastoral social de diferentes iglesias y templos de la comunidad que se visitaron para presentarles el proyecto general del cual se deriva el presente estudio. En las organizaciones donde existía un comité de ética establecido formalmente, se analizaron las consideraciones éticas y, al igual que en cada una de las organizaciones, se hicieron acuerdos de colaboración entre el equipo de investigación y la organizacióninstitución referente. Una vez instituidos, se procedió a establecer contacto con los familiares de los adultos mayores que reciben los servicios, a quienes se les dio a conocer el contenido de la Carta de Consentimiento Informado en la que se explican los objetivos del proyecto y su proceder general, invitándoles a participar de manera voluntaria.

Se consideró contacto por redes personales cuando los participantes fueron referenciados por personas familiarizadas (estudiantes, compañeros de trabajo) con los propósitos del proyecto. Una vez localizados, se procedió a la explicación de las características del estudio, con base en el contenido de la Carta de Consentimiento Informado y finalmente se extendió la invitación para la participación voluntaria.

Con los participantes que aceptaron tomar parte del estudio se programaron visitas domiciliarias para obtener el total de la información. A partir del número de instrumentos de recolección, se programó que la aplicación de los mismos se llevara a cabo en un lapso de dos a cinco sesiones de colecta para cada participante La duración promedio para cada sesión fue de una hora.

Como parte de la estrategia de aplicación para la optimización del tiempo, se promovió que los aplicadores realizaran, a manera de entrevista estructurada, el cuestionario sobre datos sociodemográficos y los del índice de dependencia funcional. Asimismo, conveniente al diseño de la Escala de Apoyo Social en la que se facilita su realización sin necesidad de dirección por parte del entrevistador, se ofreció la posibilidad de responderse de manera independiente por los cuidadores que así lo prefirieron o bien asistidos por el entrevistador por quienes lo solicitaron. El tiempo para diligenciar la escala duró en promedio 10 minutos.

\section{Resultados}

Previo a la validación de la escala, se realizó un conjunto de análisis para examinar el cumplimiento de presupuestos multivariados en el banco de datos, atendiendo a los procedimientos sugeridos por Tabachnick y Fidell (2007). Con un análisis de estadística descriptiva se identificó que ninguno de los ítems a validar tenía un porcentaje de valores perdidos mayor a $5 \%$, descartando así la posibilidad de la improcedencia del formato de los ítems. También se descartó la anormalidad de las distribuciones de los ítems en virtud de los valores de asimetría menores a \pm 1 . Por otro lado, se asumió la ausencia de multicolinialidad, pues ninguno de los ítems obtuvo valores de tolerancia menores a 0.3 , indicando que la correlación múltiple cuadrada entre variables no implicaba situaciones de singularidad entre los ítems. Posteriormente, se identificaron casos discrepantes multivariados (outliers) mediante los valores de distancia de Mahalanobis. Comparando los resultados con una distribución de $\mathrm{X}^{2}[(23)=35.17 ; p \leq 0.01]$, se encontraron 43 casos discrepantes, los cuales se eliminaron.

Una vez revisado el cumplimiento básico de presupuestos multivariados en el conjunto de datos, se verificó el número de participantes (222) en re- 
TABLA 2

Análisis factorial (método de ejes principales) de la EAP/CFAM

\begin{tabular}{|c|c|c|c|c|}
\hline $\begin{array}{c}\text { Ítem } \\
\text { Cuando usted lo necesita, recibe apoyo para... }\end{array}$ & F1 & $\mathrm{F} 2$ & F3 & $\mathrm{F} 4$ \\
\hline Realizar los quehaceres de la casa & 0.830 & & & \\
\hline Preparar la comida & 0.826 & & & \\
\hline Cuidar de su propia salud & 0.759 & & & \\
\hline Cuidar de su familiar adulto mayor & 0.739 & & & \\
\hline Atender a los otros miembros de la familia & 0.716 & & & \\
\hline Ir por el mandado & 0.697 & & & \\
\hline Llevarla/o a los lugares a los que necesita ir & 0.547 & & & \\
\hline Pagar lo gastos diarios & & 0.928 & & \\
\hline Conseguir o comprar lo necesario para preparar la comida & & 0.919 & & \\
\hline Pagar los gastos de la casa, como el agua, la luz, el teléfono, etc. & & 0.912 & & \\
\hline Pagar gastos médicos, medicamentos, tratamientos o terapias & & 0.814 & & \\
\hline Pagar gastos repentinos o de emergencia & & 0.774 & & \\
\hline Recibir apoyo moral o emocional si se siente triste & & & 0.863 & \\
\hline Hablar sobre sus dudas o miedos sin sentirse criticado/a & & & 0.842 & \\
\hline Desahogarse cuando tiene problemas o preocupaciones & & & 0.809 & \\
\hline Tiene con quien compartir sus alegrías, sus planes, sus sueños & & & 0.777 & \\
\hline Recibir compañía o cariño & & & 0.726 & \\
\hline Alguien reconoce su esfuerzo al cuidar a su familiar adulto mayor & & & 0.507 & \\
\hline Saber cómo atender a los demás miembros de la familia & & & & 0.794 \\
\hline Resolver problemas familiares que van surgiendo & & & & 0.786 \\
\hline Saber cómo cuidar de su propia salud & & & & 0.774 \\
\hline Saber cómo cuidar de la salud o prevenir enfermedades & & & & 0.715 \\
\hline Saber qué hacer en emergencias o situaciones imprevistas & & & & 0.648 \\
\hline F1.- Percepción de apoyo práctico & 1.00 & & & \\
\hline F2.- Percepción de apoyo material & 0.435 & 1.00 & & \\
\hline F3.- Percepción de apoyo emocional & 0.424 & 0.439 & 1.00 & \\
\hline F4.- Percepción de apoyo de orientación & 0.614 & 0.354 & 0.583 & 1.00 \\
\hline Alfa de Cronbach por factor & 0.93 & 0.95 & 0.91 & 0.89 \\
\hline Alfa total del instrumento & \multicolumn{4}{|c|}{0.95} \\
\hline
\end{tabular}

Fuente: elaboración propia.

lación con el número de variables a factorizar (23), encontrándose que para este estudio se cuenta con 9.6 casos por cada ítem que se va a factorizar.

Para examinar la factorabilidad de la matriz de datos se observaron los siguientes indicadores: a) $\mathrm{KMO}=0.91$; b) un valor de $0.001 \mathrm{co-}$ mo determinante de la matriz y c) un valor de $\mathrm{X}^{2}=2316 ; p \leq 0.001$ en el Test de Esfericidad de Bartlett. Con las anteriores evidencias se procedió entonces a realizar los análisis correspondientes para evaluar las propiedades psicométricas de la medida de apoyo social.
Se realizaron análisis factoriales por ejes principales, extrayendo cuatro factores que explican $63 \%$ de la varianza del constructo apoyo social coherente teóricamente y con propiedades psicométricas muy satisfactorias. Se utilizó la rotación PROMAX dada la alta correlación entre los dos factores. En la Tabla 2 se exponen los resultados.

La EAP/CFAM mostró índices robustos de validez y confiabilidad, confirmando una estructura de factores teóricamente coherente. Posteriormente, se realizaron análisis de correlación $r$ de Pearson entre las puntuaciones factoriales de apoyo social 
TABLA 3

Correlación de Pearson

\begin{tabular}{lcccc}
\hline & Apoyo material & Apoyo práctico & $\begin{array}{c}\text { Apoyo } \\
\text { emocional }\end{array}$ & $\begin{array}{c}\text { Apoyo de } \\
\text { orientación }\end{array}$ \\
\hline Satisfacción con apoyo material & $0.506^{* *}$ & $0.347^{* *}$ & $0.370^{* *}$ & $0.315^{* * *}$ \\
Satisfacción con apoyo práctico & $0.29 * *$ & $0.468^{* *}$ & $0.431^{* *}$ & $0.300^{* *}$ \\
Satisfacción con apoyo emocional & $0.212^{* *}$ & $0.206^{* *}$ & $0.646^{* *}$ & $0.372^{* *}$ \\
Satisfacción con apoyo de orientación & $0.182^{* *}$ & $0.257^{* *}$ & $0.435^{* *}$ & $0.521^{* *}$ \\
Tamaño de la red social & $0.122^{*}$ & $0.204^{* *}$ & $0.168^{* *}$ & $0.167^{* *}$ \\
\hline
\end{tabular}

Fuente: elaboración propia.

y las puntuaciones de satisfacción con el apoyo en cada factor y el tamaño de la red social (Tabla 3).

Las correlaciones entre la percepción de apoyo y la satisfacción con el apoyo en el factor correspondiente mostraron valores positivos, significativos e indicativos de valores de asociación medios y medio/alto. Por su parte, el tamaño de la red social arrojó valores de correlación bajos con las cuatro puntuaciones factoriales.

Enseguida, se corrieron análisis de ecuaciones estructurales para confirmar la estructura de factorial previamente identificada. Los resultados mostraron que los cuatro factores representan adecuadamente a la variable latente apoyo social. Los índices de ajuste del modelo de medida fueron: $\chi^{2}(1.249)=1.41, p \geq 0.05 ; \mathrm{GFI}=0.99, \mathrm{NFI}=0.99$, $\mathrm{CFI}=0.99$; $\mathrm{AGFI}=0.97$; $\mathrm{RMSEA}=0.041$ (intervalo de confianza de $90 \%=0.000$ a 0.18). Así, se demostró que el apoyo social tiene una relación causal positiva y significativa con Apoyo Material $(\beta=0.66)$, Apoyo Práctico $(\beta=0.69)$, Apoyo Emocional $(\beta=0.60)$ y Apoyo De Orientación $(\beta=0.81)$; se observó también la covarianza de -0.43 entre los errores de medida de los factores Apoyo Material y Apoyo de Orientación. De acuerdo a la lógica de las ecuaciones estructurales, la magnitud de los pesos factoriales sugiere una fuerte relación de la variable latente (apoyo social) con sus cuatro indicadores (Figura 1).

\section{Discusión}

Dado que los familiares proveen cuidado a sus mayores dependientes, ellos necesitan apoyo que pueda subsanar el deterioro que pueden llegar a sentir (Marziali et al., 2010). Esto es especialmente importante si se considera que al menos la mitad de la población tiene posibilidades de convertirse en cuidador, al menos durante un período de su vida (Lloyd, 1996 citado en Luxardo, 2009).

Ante la falta de medidas de apoyo social para ser aplicables a cuidadores familiares en México, prácticas de investigación han consistido en utilizar preguntas dicotómicas o politómicas generales sobre el tema pero sin sustento psicométrico (ej., Lara, González \& Blanco, 2008), que producen resultados que poco contribuyen al conocimiento de la problemática en nuestro país, situación que se da con el agravante de la poca actividad académica que en nuestro país se genera al respecto. La falta de conocimiento sólido sobre las necesidades que viven los cuidadores familiares es una condición más que los vulnera, pues perpetúa la dinámica actual en la que el cuidador se convierte en un paciente oculto, en un asistente desasistido.

La EAP/CFAM mostró índices de validez y confiabilidad muy satisfactorios para dar cuenta del apoyo percibido por cuidadores, tanto en análisis exploratorios como confirmatorios; se obtuvo que los cuatro factores de la escala explican $63 \%$ de la varianza del constructo, integrando 23 ítems con cargas factoriales de 0.51 a 0.93 y los valores de consistencia interna de los factores y de la escala total que oscilan entre 0.85 y 0.95 . A nivel confirmatorio, se demostró que el apoyo social tiene una relación causal positiva y significativa con Apoyo Material $(\beta=0.66)$, Apoyo Práctico $(\beta=0.69)$, Apoyo Emocional $(\beta=0.60)$ y Apoyo de Orientación $(\beta=0.81)$, con valores de ajuste del modelo $\geq 0.97$ y en RMSEA de 0.041 . 

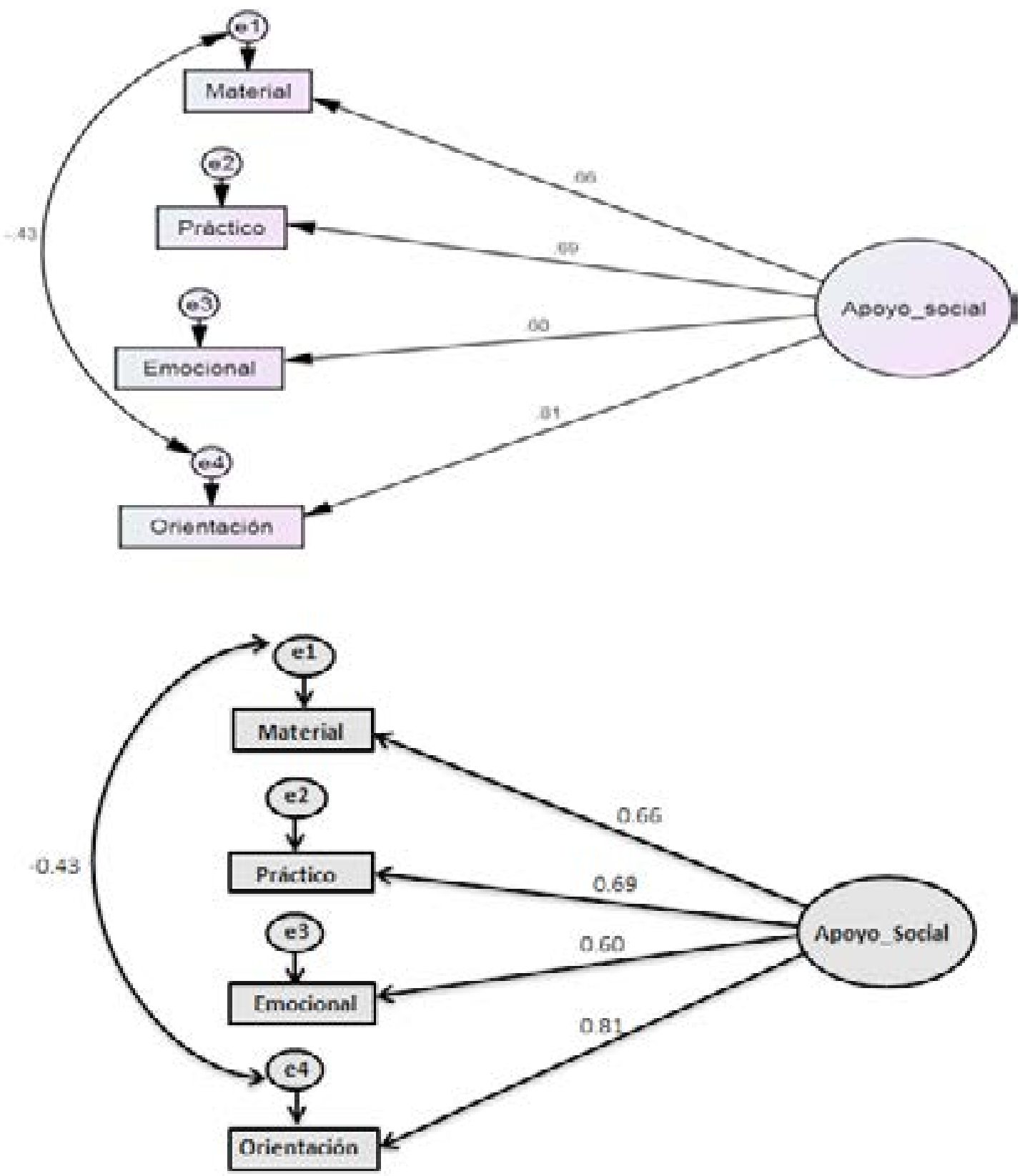

Figura 1. Valores de regresión estandarizados para los cuatro factores de la AP/CFAM.

Fuente: elaboración propia.

Otros instrumentos que han sido diseñados para población general pero que en la literatura se reporta haber sido utilizados en estudios de cuidadores son: (a) el Social Support Questionnaire ([SSQ]; Saranson, Saranson, Shearin \& Pierce, 1987) utilizado, por ejemplo, por Hudson y Hayman-White
(2006), obteniendo las siguientes propiedades psicométricas en su muestra de cuidadores: $67.3 \%$ de la varianza explicada, con cargas factoriales de 0.75 a 0.9 y valor de alfa de Cronbach de 0.9 y (b) Wilks (2009) empleó la Social Support Scale ([PSSS]; Procidano \& Heller, 1983) en su versión abreviada 
hecha en población general por Maton et al. (1996). Wilks hizo la validación en una muestra de cuidadores de adultos mayores obteniendo una solución factorial que explicó el $74 \%$ en una submuestra y $76 \%$ en una segunda submuestra, con valores de consistencia interna de 0.89 y 0.9 .

Una ventaja que ofrece la EAP/CFAM medida frente a otras escalas de apoyo que fueron desarrolladas para población general, es que ésta se centra en las necesidades que estudios previos han demostrado ser relevantes para cuidadores familiares de adultos mayores en contextos socioculturales latinoamericanos (Brasil y México), y que muestran que el cuidador convive con una multiplicidad de demandas derivadas de sus diferentes roles sociales (ej., mamá/papá, hija/hijo, hermana/hermano, trabajadora/trabajador) que le confrontan en cuestiones económicas (para los gastos cotidianos y relativos a la salud), prácticas (actividades instrumentales que le permitan cuidar al adulto mayor pero también continuar atendiendo las labores domésticas), emocionales (reconocimiento de la labor como cuidador e posibilidad de explayar sus emociones positivas y negativas) y de información que retroalimente decisiones que el cuidador necesita tomar tanto para cuidador del adulto mayor, de los demás miembros de la familia y de sí mismo (Domínguez-Guedea, Damacena et al., 2009; Domínguez-Guedea, Tróccoli et al., 2009). Es esta una realidad compleja que vive el cuidador y que quedó apropiadamente representada en los cuatro factores de la EAP/CFAM (Apoyo Material, Práctico, Emocional y de Orientación), con un muy porcentaje de explicación del constructo apoyo social (63\%).

El enfoque de la EAP/CFAM en las demandas particularmente compartidas por cuidadores familiares latinoamericanos, es importante a la luz de la hipótesis de la especificidad del apoyo que la literatura clásica indica para explicar que los efectos benéficos de las relaciones interpersonales operaran bajo circunstancias específicas y que correspondan con las demandas particulares del estresor o de la experiencia estresante (Cohen \& McKay, 1984). En este sentido, los datos producidos por la EAP/ CFAM pueden ser más sensibles para captar la aportación que el apoyo social puede hacer en modelos conceptuales sobre el bienestar en cuidadores familiares de adultos mayores, así como para diseñar acciones de apoyo para esta población vulnerable.

La EAP/CFAM se constituye entonces como una medida válida y confiable que permite identificar el apoyo social percibido por cuidadores familiares de adultos mayores. Esta es una herramienta que puede ser útil para ser aplicada a gran escala y así dimensionar parte de un importante problema social que se vive en México, pero que, desafortunadamente, ha pasado inadvertido en la actual política mexicana de envejecimiento, salud y desarrollo social. La invisibilidad de la figura del cuidador en nuestro contexto se mantiene en una inercia conveniente para el sistema público pero desgastante para las familias de los adultos mayores con problemas de salud y es que, entre mejor haga su trabajo el cuidador en el ambiente doméstico, las instituciones ahorran costos de hospitalización, recurso humano y días de cama (Márquez-González et al., 2010).

En la consideración de que lo que no se mide difícilmente podrá ser mejorado, se propone el uso de la EAP/CFAM junto con otras medidas que permitan valorar las necesidades que enfrentan las familias, para así generar acciones de apoyo eficientes dirigidas no solo al adulto-mayor, sino también a quien lo cuida.

\section{Referencias}

Arango-Lasprilla, J. C., Moreno, A., Rogers, H. \& Francis, K. (2009). The effect of dementia patient's physical, cognitive, and emotional/behavioral problems on caregiver well-being: Findings from a Spanish-speaking sample from Colombia, South America. American Journal of Alzheimer's Disease Eु Other Dementias, 24(5), 384-395. doi: 10.1177/1533317509341465

Barrón, A. \& Sánchez, E. (2001). Estructura social, apoyo social y salud mental. Psicothema, 13(1), 17-23.

Betrabet, G. (2009). Restorative home environments for family caregivers. Journal of Aging Studies, 23(3), 197-204. doi: 10.1016/j.jaging.2007.11.005 
Cohen, S. \& McKay, G. (1984). Social support, stress and the buffering hypothesis: A theoretical analysis. In A. Baum, S. E. Taylor \& J. E. Singer (Eds.), Handbook of psychology and health (Vol. 4, pp. 253 . 267). Hillsdale, NJ: Erlbaum.

Crespo, M. \& López, J. (2007). El apoyo a los cuidadores de familiares mayores dependientes en el hogar: desarrollo del programa "Cómo mantener su bienestar". Madrid: Ministerio de Trabajo y Asuntos Sociales, Secretaría de Estado de Servicios Sociales, Familias y Discapacidad, Instituto de Mayores y Servicios Sociales (IMSERSO).

Domínguez-Guedea, M. (2005). Modelo de estrés y bienestar subjetivo en cuidadores de familiares de adultos mayores dependientes funcionales. Tesis de doctorado inédita, Universidade de Brasilia, Brasil.

Domínguez-Guedea, M., Damacena, F., Montiel, M. M., Ochoa, P., Álvarez, G., Valdéz, L., et al. (2009). Necessidades de apoio social em cuidadores de familiares idosos mexicanos. Psicologia E3 Sociedade, 21(2), 242-249. doi: 10.1590/S0102. 71822009000200011

Domínguez-Guedea, M. \& López-Dávalos, G. (2008). Bienestar de cuidadores familiares de adultos mayores. Sonárida, 12(26), 13-16.

Domínguez-Guedea, M., Tróccoli, B., Karino, C., Ciancio, J. C., Hernández, D. \& Pantoja, C. E. (2009). Apoyo social en cuidadores de familiares de personas adultas mayores con problemas de salud. En F. Cintrón, E. Acosta \& L. Díaz (Eds.), Psicología comunitaria: trabajando con comunidades en las Américas (pp. 191-209). Hatorey, Puerto Rico: Publicaciones Puertorriqueñas.

Fernández-Lansac, V. \& Crespo, M. (2011). Resiliencia, personalidad resistente y crecimiento en cuidadores de personas con demencia en el entorno familiar: una revisión. Clínica y Salud, 22(1), 21-40.

Gallegos-Carrillo, K., Mudgal, J., Sánchez-García, S., Wagner, F. A., Gallo, J. J., Salmerón, J., et al. (2009). Social networks and health-related quality of life: A population based study among older adults. Salud Pública de México, 51(1), 6-13. doi: 10.1590/S0036-36342009000100004

Golden, J., Conroy, R. M., Bruce, I., Denihan, A., Greene, E., Kirby, M., et al. (2009). Loneliness, social support networks, mood and wellbeing in community-dwelling elderly. International Journal of Geriatric Psychiatry, 24(7), 694-700. doi: 10.1002/ gps.2181

Gracia, E. \& Herrero, J. (2006). La comunidad como fuente de apoyo social: evaluación e implicaciones en los ámbitos individual y comunitario. Revista Latinoamericana de Psicología, 38(2), 327-342.

Gutiérrez-Robledo, L. M., Ávila-Fematt, F. M. \& Montaña-Álvarez, M. (2010). La geriatría en México. El Residente, 5(2), 43-48.

Guzmán, J. M., Huenchuan, S. \& Montes de Oca, V. (2003, julio). Redes de apoyo social de personas mayores: marco teórico conceptual. En Viejos y Viejas. Participación, ciudadanía e inclusión social. Simposio llevado a cabo en el 51 Congreso Internacional de Americanistas. Comisión Económica para América Latina y el Caribe/División de Población de la CEPAL, Santiago de Chile, Chile.

Hudson, P. L. \& Hayman-White, K. (2006). Measuring the psychosocial characteristics of family caregivers of palliative care patients: Psychometric properties of nine self-report instruments. Journal of Pain and Symptom Management, 31(3), 215-228. doi: 10.1016/j.jpainsymman.2005.07.010

Huenchuan, S. \& Guzmán, J. M. (2007). Políticas hacia las familias con personas mayores: el desafío del cuidado en la edad avanzada. En I. Arriagada (Coord.), Familias y politicas públicas en América Latina: una historia de desencuentros (pp. 273-293). Santiago de Chile: Naciones Unidas.

Hupcey, J. E. (1998). Clarifying the social support theory-research linkage. Journal of Advanced Nursing, 27(6), 1231-1241.

Kafetsios, K. \& Sideridis, G. D. (2006). Attachment, social support and well-being in young and older adults. Journal of Health Psychology, 11(6), 863-876. doi: 10.1177/1359105306069084

Lara, G., González, A. \& Blanco, L. A. (2008). Perfil del cuidador: sobrecarga y apoyo familiar e institucional del cuidador primario en el primer nivel de atención. Revista de Especialidades MédicoQuirúrgicas, 13(4), 159-66. Recuperado de http:// www.nietoeditores.com.mx/download/especialidades mq/2008/Oct-Dic/RevEspMQ-159-66.pdf

Lavela, S. L. \& Ather, N. (2010). Psychological health in older adult spousal caregivers of 
older adults. Chronic Illness, 6(1), 67-80. doi: 10.1177/1742395309356943

Lawton, M. P. \& Brody, E. M. (1969). Assessment of older people: Self-maintenance and instrumental activities of daily living. The Gerontologist, 9(3), 179-186.

Lee, M. (2008). Caregiver stress and elder abuse among Korean family caregivers of older adults with disabilities. Journal of Family Violence, 23(8), 707-712. doi: 10.1007/s10896-008-9195-2

Lin, N. \& Ensel, W. (1989). Life stress and health: Stressors and resources. American Sociological Review, 54(3), 382-399.

Lizárraga, V., Artetxe, I. \& Posua, N. (2008). Eficacia de una intervención psicológica a domicilio dirigida a personas cuidadoras de mayores dependientes. Revista Española de Geriatría y Gerontología, 43(4), 229-234.

Luxardo, N. (2009). Políticas públicas para cuidadores informales. Savia, 7, 23-26.

Mahoney, F. I. \& Barthel, D. W. (1965). Functional evaluation: The Barthel Index. Maryland Medical Journal, 14, 61-65.

Márquez-González, M., López, J., Romero-Moreno, R. \& Losada, A. (2010). Anger, spiritual meaning and support from the religious community in dementia caregiving. Journal of Religion $\mathcal{E}$ Health. Publicación anticipada en línea. doi: 10.1007/ s10943-010-9362-7

Marziali, E., McCleary, L. \& Streiner, D. L. (2010). Evaluation of an assessment battery for estimating dementia caregiver needs for health and social care services. American Journal of Alzheimer's Disease and Other Dementians, 25(5), 446-454. doi: 10.1177/1533317510370958

Maton, K. I., Teti, D. M., Corns, K. M., Vieira-Baker, C. C., Lavine, J. R., Gouze, K. R., et al. (1996). Cultural specificity of support sources, correlates and contexts: Three studies of African-American and Caucasian youth. American Journal of Community Psychology, 24(4), 551-587.

Méndez, M. J. \& Pozo, C. (2011). Apoyo funcional vs. disfuncional en una muestra de pacientes crónicos. Su incidencia sobre la salud y el cumplimiento terapéutico. Anales de Psicología, 27(1), 47-57.
Molina, J. M., Iáñez, M. \& Iáñez, B. (2005). El apoyo social como modulador de la carga del cuidador de enfermos de Alzheimer. Psicología y Salud, 15(1), 33-43.

Monahan, D. J. \& Hooker, J. (1997). Caregiving and social support in two illness groups. Social Work, 42(3), 278-287.

Ostwald, S. K. (2009). Who is caring for the caregiver? Family Community Health, 32(1S), S5-S14.

Pearce, M. J. \& Singer, J. L. (2006). Religious coping among caregivers of terminally ill cancer patients. Journal of Health Psychology, 11(5), 743-759. doi: 10.1177/1359105306066629

Procidano, M. E. \& Heller, K. (1983). Measures of perceived social support from friends and from family: Three validation studies. American Journal of Community Psychology, 11(1), 1-24.

Rapp, S. R., Shumaker, S., Schmidt, S., Naughton, M. \& Anderson, R. (1998). Social resourcefulness: Its relationship to social support and wellbeing among caregivers of dementia victims. Aging $\mathbb{E}$ Mental Health, 2(1), 40-48. doi: 10.1080/13607869856920

Reid, R. C., Stajduhar, K. I. \& Chappell, N. L. (2010). The impact of work interferences on family caregiver outcomes. Journal of Applied Gerontology, 29(3), 267-289. doi: 10.1177/0733464809339591

Rodríguez-Agudelo, Y., Mondragón-Maya, A., Paz-Rodríguez, F., Chávez-Oliveros, M. \& Solís-Vivanco, R. (2010). Variables asociadas con ansiedad y depresión en cuidadores de pacientes con enfermedades neurodegenerativas. Archivos de Neurociencias, 15(1), 25-30.

Saranson, I. G., Saranson, B. R., Shearin, E. N. \& Pierce, G. R. (1987). A brief measure of social support: Practical and theoretical implications. Journal of Social and Personal Relationships, 4(4), 497-510.

Shah, S., Vanclay, F. \& Cooper, B. (1989). Improving the sensitivity of the Barthel Index for stroke rehabilitation. Journal of Clinic Epidemiology, 42(8), 703 709.

Spaid, W. M. \& Barusch, A. (1994). Emotional closeness and caregiver burden in the marital relationship. Journal of Gerontological Social Work, 21(3), 197212. doi: 10.1300/J083V21N03 13

Spijker, A., Verhey, F., Graff, M., Grol, R., Adang, E., Wollersheim, H., et al. (2009). Systematic care for 
caregivers of people with dementia in the ambulatory mental health service: Designing a multicentre, cluster, randomized, controlled trial. BMC Geriatrics, 9(21). doi: 10.1186/1471-2318-9-21

Steiner, V., Pierce, L., Drahuschak, S., Nofziger, E., Buchman, D. \& Szirony, T. (2008). Emotional support, physical help, and health of caregivers of stroke survivors. Journal of Neuroscience Nursing, 40(1), 48-54.

Tabachnick, B. G. \& Findell, L. S. (2007). Using multivariate statics. Boston: Pearson Education.
Úbeda, I. \& Roca, M. (2008). Los cuidados familiares. Nursing, 26(9), 56-59.

Van Den Wijngaart, M. A. G., Vernooij-Dassen, M. J. F. J. \& Felling, A. J. A. (2007). The influence of stressors, appraisal and personal conditions on the burden of spousal caregivers of persons with dementia. Aging $\mathcal{E}$ Mental Health, 11(6), 626-636. doi: $10.1080 / 13607860701368463$

Wilks, S. E. (2009). Support for Alzheimer's caregivers: Psychometric evaluation of familial and friend support measures. Research on Social Work Practice, 19(6), 722-729. doi: 10.1177/1049731508329404 